\title{
Second-harmonic imaging of poled silica waveguides
}

Arentoft, Jesper; Pedersen, Kjeld; Bozhevolnyi, Sergey I.; Kristensen, Martin; Yu, Ping; Nielsen, Christian Bergenstof

Published in:

Applied Physics Letters

Link to article, DOI:

$10.1063 / 1.125644$

Publication date:

2000

Document Version

Publisher's PDF, also known as Version of record

Link back to DTU Orbit

Citation $(A P A)$ :

Arentoft, J., Pedersen, K., Bozhevolnyi, S. I., Kristensen, M., Yu, P., \& Nielsen, C. B. (2000). Second-harmonic imaging of poled silica waveguides. Applied Physics Letters, 76(1), 25-27. https://doi.org/10.1063/1.125644

\section{General rights}

Copyright and moral rights for the publications made accessible in the public portal are retained by the authors and/or other copyright owners and it is a condition of accessing publications that users recognise and abide by the legal requirements associated with these rights.

- Users may download and print one copy of any publication from the public portal for the purpose of private study or research.

- You may not further distribute the material or use it for any profit-making activity or commercial gain

- You may freely distribute the URL identifying the publication in the public portal

If you believe that this document breaches copyright please contact us providing details, and we will remove access to the work immediately and investigate your claim 


\title{
Second-harmonic imaging of poled silica waveguides
}

\author{
Jesper Arentoft ${ }^{\mathrm{a})}$ \\ Research Center COM, DTU Bldg. 349, DK-2800 Lyngby, Denmark \\ Kjeld Pedersen and Sergey I. Bozhevolnyi \\ Institute of Physics, Aalborg University, Pontoppidanstrcede 103, DK-9220 Aalborg, Denmark \\ Martin Kristensen and Ping Yu \\ Research Center COM, DTU Bldg. 349, DK-2800 Lyngby, Denmark
}

Christian B. Nielsen

Mikroelektronik Centret, DTU Bldg. 345east, DK-2800 Lyngby, Denmark

(Received 3 May 1999; accepted for publication 10 November 1999)

\begin{abstract}
Electric-field poled silica-based waveguides are characterized by measurements of second-harmonic generation (SHG) and of the linear electro-optic effect (LEO). A SHG scanning technique allowing for high-resolution imaging of poled devices is demonstrated. Scans along the direction of the poling field show that the second-order optical nonlinearity is located near the interface between differently doped glass layers. Both SHG and LEO measurements indicate that the ratio between the main elements of the second-order nonlinear optical susceptibility tensor, $\chi_{33}^{(2)}$ and $\chi_{31}^{(2)}$, is significantly smaller than three. (C) 2000 American Institute of Physics. [S0003-6951(00)03301-5]
\end{abstract}

Poled silica glass is an attractive, but not yet fully developed material, for photonic applications, e.g., electro-optic modulation and wavelength conversion. There is a growing effort in the development of glasses possessing large and stable second-order optical nonlinearities (SON). ${ }^{1-5}$ Secondharmonic generation (SHG) and interferometric measurements of the linear electro-optic (LEO) effect are the most widely used techniques for probing of SON. Tools for the examination of spatial variations of SON are highly valuable in studies aiming at understanding the fundamental mechanisms of poling as well as for characterization of poled components, e.g., wavelength conversion devices based on quasiphase matching obtained by periodic poling. SHG microscopy has successfully been applied for investigations of SON in periodically poled ferroelectric crystals, ${ }^{6}$ domain structures in magnetic garnet films, ${ }^{7}$ and ferroelectric domain walls. ${ }^{8}$ In this letter, we present spatially resolved measurements of SHG in poled silica-based waveguides along with the conventional characterization of the LEO effect in these waveguides. Two sets of experiments were carried out in order to explore the potential of the SHG imaging technique.

Three layers of glass were deposited by plasma enhanced chemical vapor deposition on a $n$-type doped silicon wafer (Fig. 1). Al was evaporated on top of the glass and the wafer was cleaved into samples of $\sim 2.5 \times 2.5 \mathrm{~cm}^{2}$. One of the samples was placed on a heater and brought to $400{ }^{\circ} \mathrm{C}$. The sample was poled by applying $+2 \mathrm{kV}$ to the Al layer and keeping the silicon wafer grounded. Before removing the high voltage, the sample was cooled to room temperature. The Al layer was removed and the sample was cleaved through the poled region. SHG microscopy was employed in order to reveal the profile of the induced SON along the coordinate parallel to the poling field (along $y$ ). The linearly polarized (pump) beam from a mode-locked Ti-sapphire la-

${ }^{a}$ Electronic mail: ja@com.dtu.dk ser $\left(\lambda_{\omega} \cong 910 \mathrm{~nm}, P_{\omega}^{\text {av }} \sim 17 \mathrm{~mW}\right.$, pulse duration $\left.\tau \sim 200 \mathrm{fs}\right)$ was focused onto the end facet of the sample with normal incidence (Fig. 1). The pump was scanned along the $y$ coordinate in steps of $\sim 0.3 \mu \mathrm{m}$. The reflected SHG signal was detected with a photomultiplier and recorded as a function of the scanning coordinate. The setup allows one to map the detected SHG signal for all combinations of $s$ and $p$ polarizations, where $s$ and $p$ denote polarizations perpendicular and parallel to the $y$ axis, respectively. $s-p$ configuration means that the pump beam is polarized along the $z$ axis whereas the detected component of the SHG field is parallel to the $y$ axis (Fig. 1).

In order to be able to measure the LEO effect, waveguides were ultraviolet (UV) induced in one of the other samples, as described in the following. Grooves of width varying from 4 to $10 \mu \mathrm{m}$ and with a spacing of 250 $\mu \mathrm{m}$ were photolitographically defined in the $\mathrm{Al}$ layer (along the $z$ coordinate of Fig. 1). The sample was subsequently exposed to UV laser radiation $(248 \mathrm{~nm})$ in order to write channel waveguides in the central photosensitive glass layer. ${ }^{9,10}$ Then, the $\mathrm{Al}$ mask was removed and replaced by a homogeneous Al layer. Finally, the sample was poled under the same conditions as described for the first sample.

Figure 2 shows the measured reflected fundamental and second-harmonic signals as functions of sample depth $(y$ co-

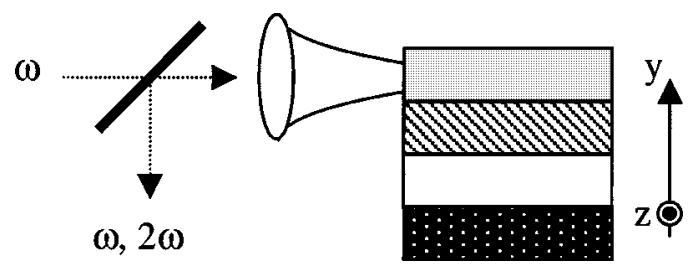

FIG. 1. Setup for scanning of SHG along the direction of the poling field. White dots on black: $\mathrm{Si}$ wafer. White: $\mathrm{SiO}_{2}$ (Thickness: $3.30 \pm 0.05 \mu \mathrm{m}$ ). Hatched: Ge:SiON (Thickness: $1.93 \pm 0.05 \mu \mathrm{m}$ ). Gray: $\mathrm{SiO}_{2}$ (Thickness: $2.69 \pm 0.05 \mu \mathrm{m})$. 


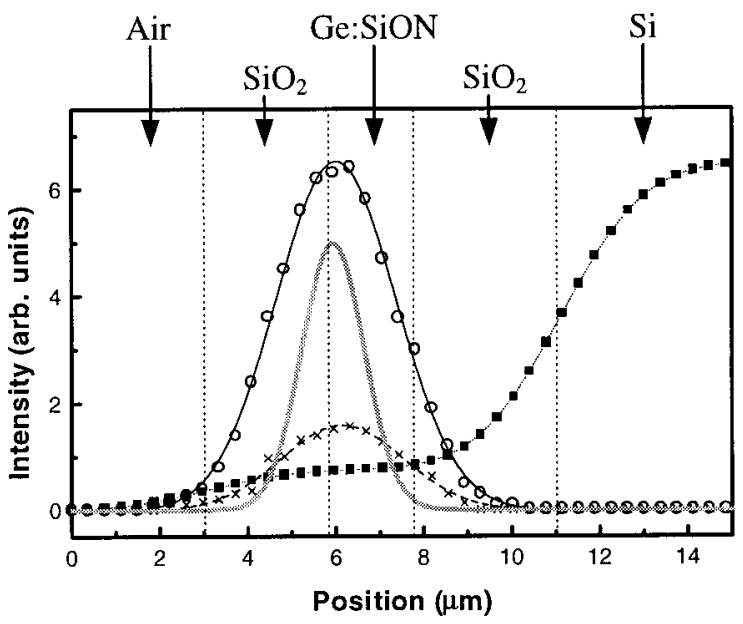

FIG. 2. SHG profiles and linear reflectivity obtained by scanning along the coordinate parallel to the poling field. Linear reflectivity (squares: experiment, dotted line: Gaussian convolution fit), SHG signal in $p-p$ configuration (circles: experiment, full line: Gaussian fit), SHG signal in $s-p$ configuration (crosses: experiment, dashed line: Gaussian fit), suggested distribution of second-order optical nonlinearity as explained in text (gray).

ordinate). Only the $p-p$ and $s-p$ configurations gave a nonzero SHG signal, as expected for homogeneously poled isotropic media. A fundamental beam width $(2 \sigma)$ of 4.6 $\pm 0.3 \mu \mathrm{m}$ was found from a convolution fit of the data points pertaining to the reflected fundamental signal, assuming a Gaussian beam profile and using step functions for the reflectivities of air, glass, and Si. A total glass film thickness of $7.93 \pm 0.06 \mu \mathrm{m}$ was also estimated from the convolution fit, while the relative thickness of the individual glass layers were estimated from scanning electron microscope data, leading to the absolute values given in the caption of Fig. 1 . From a Gaussian fit of the SHG data (also shown in Fig. 2) it is found that the SON is at its maximum at $2.94 \pm 0.10 \mu \mathrm{m}$ from the sample surface. The SON peak corresponds, except for a few tens of a micrometer, to the position of the interface between core and top cladding layers of the sample. The thickness of the SON layer is estimated by deconvoluting the SHG data, assuming a Gaussian SON profile and the fundamental beamwidth found earlier. From this a thickness of $2.0 \pm 0.5 \mu \mathrm{m}$ is obtained, that is, the SON decays to $1 / e$ only $\sim 1 \mu \mathrm{m}$ away from each side of the interface. The suggested SON profile is shown in Fig. 2.

In order to estimate absolute values of the SHG signals, the setup was calibrated by measuring $\chi_{123}^{(2)}$ of a GaAs crystal under the same experimental conditions. In the $p-p$ configuration, the SHG signal for GaAs was found to be about four orders of magnitude larger than the peak value of the SHG signal for the poled silica sample. This signal level corresponds to $\chi_{33}^{(2)} \sim 0.5 \mathrm{pm} / \mathrm{V}$ for the poled silica. All of the UVwritten waveguides in the thermally poled sample showed a LEO coefficient of $\sim 0.045 \mathrm{pm} / \mathrm{V}$, as measured with a fiberbased Mach-Zehnder interferometer (MZI), described in Ref. 5. Having in mind the limited extension of the SON and a possible dispersion of $\chi^{(2)}$, the SHG and LEO data are not in disagreement.

A ratio of 3:1 between $\chi_{33}^{(2)}$ and $\chi_{31}^{(2)}$ of the SON susceptibility tensor, has been suggested to exist for homogeneously poled isotropic media under certain approximations. ${ }^{4,11}$ A ratio of $4: 1$ between the $p-p$ and $s-p$ Downloaded 31 Jan 2010 to 192.38.67.112. Redistribution subject

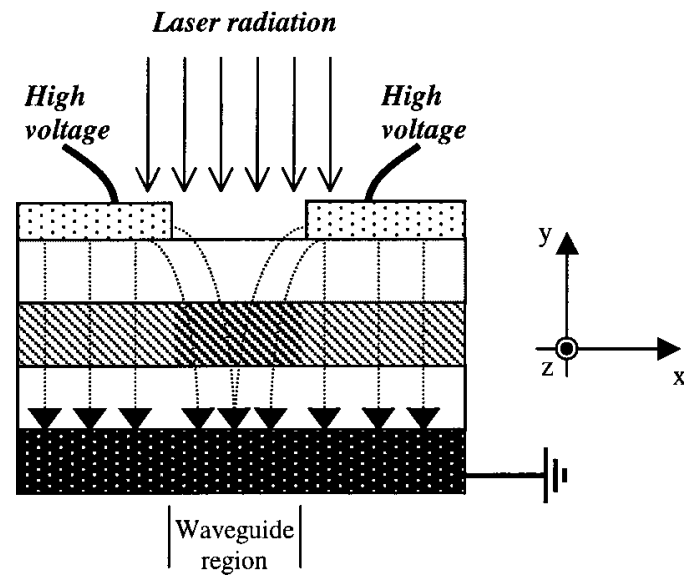

FIG. 3. Selected cross-sectional view of channel waveguide sample illustrating UV writing and radiation-assisted poling. White dots on black: Si wafer. White: $\mathrm{SiO}_{2}$. Hatched: Ge:SiON. Gray: $\mathrm{SiO}_{2}$. Bold hatched: Ge:SiON channel waveguide (along $z$ ). Black dots on white: Al electrode/ mask. Dotted arrows: distribution of the poling electric field. Plain arrows: IR radiation (during IR poling) or UV radiation (during writing of waveguides).

SHG signals was estimated from the present measurements, suggesting that $\chi_{33}^{(2)} / \chi_{31}^{(2)} \sim 2$. The ratio, $r_{\mathrm{TM}} / r_{\mathrm{TE}}$, between the electro-optic coefficients measured here for transverse magnetic and transverse electric modes, corresponding to the ratio $\chi_{33}^{(2)} / \chi_{31}^{(2)}$ (if Kleinman symmetry is assumed) yielded $1.1 \pm 0.1$. These results indicate in turn that the observed SON at least partly is due to oriented dipoles (a pure space charge effect would lead to a ratio of 3:1). ${ }^{11}$ Dispersion effects could cause the difference in the ratios observed by electro-optic modulation and SHG, respectively.

In order to explore further the potential of the SHG imaging technique we chose a sample which we expected to show considerable variations in the induced SON as a function of horizontal scanning coordinates $(x$ and $z$ ). This sample was first poled and then waveguides were UV written in it. The sample was poled by application of $1.5 \mathrm{kV}$ to the $\mathrm{Al}$ and simultaneous exposure to $\mathrm{CO}_{2}$ laser radiation (10.6 $\mu \mathrm{m})$. The silicon wafer was grounded. With this procedure the glass regions below the grooves in the $\mathrm{Al}$ mask were exposed to radiation and to a somewhat inhomogeneous electric field (Fig. 3). After poling, waveguides were written following the procedure described earlier. A voltage of $1 \mathrm{kV}$ was applied to the Al during UV exposure in order not to erase the nonlinear optical effects ${ }^{12}$ possibly induced during the infrared (IR)-poling treatment described earlier. In contrast to what has been observed by others, ${ }^{13}$ our previous experiments with similar samples showed that only very small SON effects are induced by the UV high voltage treatment alone. ${ }^{5}$ After removing the $\mathrm{Al}$, the pump beam was focused onto the sample surface under $40^{\circ}$ angle of incidence and the sample was scanned for SHG along both $x$ and $z$ coordinates. The waveguides were oriented parallel to the plane of incidence. In this setup $s-p$ configuration, for example, means that the polarization of the pump beam is parallel to the $x$ axis whereas the detected component of the SHG field is polarized in the $z-y$ plane.

The SHG measurements of this sample revealed rather complicated signals [Fig. 4(a)]. First, it is noted that SHG signals are present for the $s-s$ and $p-s$ configurations. This AlP license or copyright; see http://apl.aip.org/apl/copyright.jsp 

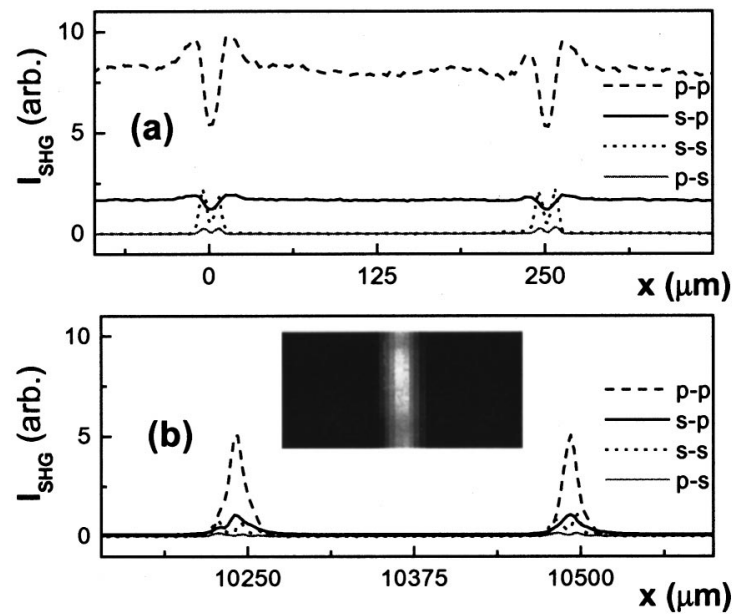

FIG. 4. (a) SHG profiles obtained by scanning along the $x$ coordinate in $p-p, s-p, p-s$ and $s-s$ polarization configurations. (b) SHG profiles and $100 \times 100 \mu \mathrm{m}^{2}$ SHG image (insert) of sample region only exposed to stray laser radiation. The profiles are obtained by scanning along $x$. The image is obtained by scanning over $x$ and $z$ coordinates in the $p-p$ polarization configuration.

observation could be attributed to the aforementioned inhomogeneity of the poling field in the waveguide regions. The widths of the peaks (in $s-s$ and $p-s$ SHG signals) and their separation agree very well with the width $(10 \mu \mathrm{m}$ in this particular region) and the spacing $(250 \mu \mathrm{m})$ of the openings in the $\mathrm{Al}$ layer. Note that, under these openings, the poling field is expected to have a component parallel to the $x$ axis (Fig. 3). Second, for the $p-p$ and $s-p$ configurations, SHG signals are observed not only in the exposed regions but also in the regions between the waveguides. This is not so surprising if one considers the IR-exposure time $(\sim 2 \mathrm{~s})$ and thermal conductance of silicon. The IR-generated heat is expected to spread substantially into the unexposed regions. For all of the polarization configurations, the SHG signals in the waveguide region show a similar double-peak structure with the dip corresponding to the waveguide center. Keeping in mind the electric field lines depicted in Fig. 3, one might suggest that the dip results from a lower poling field in the waveguide center. Alternatively, the dip could be the result of a too high poling temperature in the exposed regions. Partial erasure of the induced SON effect by UV light is yet another possible reason for this effect. ${ }^{12}$ More experiments would indeed be needed in order to clarify what mechanism causes the dip. Thus, it must be emphasized that the present data mainly serve as a demonstration of the potential of the SHG technique in characterization of poled material.
Two-dimensional SHG imaging is illustrated with the insert in Fig. 4(b), showing a $100 \times 100 \mu \mathrm{m}^{2}$ SHG image of a single waveguide region. This image was obtained in a region of the sample exposed only to stray laser radiation (IR and UV). From the SHG image and the SHG profiles shown in Fig. 4(b), it is seen that poling has taken place only in and around the waveguide region, probably because of less spreading of heat generated by IR radiation of lower intensity (stray light). The image obtained (insert) provides additional information of the SON distribution along the waveguide region. Clearly, such an imaging would be an extremely valuable tool in the development process of periodically poled silica components. All of the waveguides in this sample had a LEO coefficient of $\sim 0.02 \mathrm{pm} / \mathrm{V}$, as measured with the MZI. Also with these waveguides the ratio $\chi_{33}^{(2)} / \chi_{31}^{(2)}$ yielded $1.1 \pm 0.1$.

In summary, we have demonstrated a powerful SHGbased technique for local characterization of poled silica devices. Poled multilayer silica thin film structures were imaged by this technique and, in addition, tested for linear electro-optic effects. The SHG profiling along the direction of the poling field showed that the SON peaks at the interface between core and top cladding layers and decays to $1 / e$ only $\sim 1 \mu \mathrm{m}$ away from each side of the interface. A ratio less than three was observed between $\chi_{33}^{(2)}$ and $\chi_{31}^{(2)}$. Finally, detailed features in the horizontal SON distribution of a sample with a complicated poling history were clearly imaged.

${ }^{1}$ D. Wong, W. Xu, S. Fleming, M. Janos, and K.-M. Lo, Opt. Fiber Technol.: Mater., Devices Syst. 5, 235 (1999).

${ }^{2}$ D. Pureur, A. C. Liu, M. J. F. Digonnet, and G. S. Kino, Opt. Lett. 23, 588 (1998).

${ }^{3}$ R. A. Myers, X. Long, and S. R. J. Brueck, Proc. SPIE 2289, 98 (1994).

${ }^{4}$ P. G. Kazansky and P. St. J. Russel, Opt. Commun. 110, 611 (1994).

${ }^{5}$ J. Arentoft, M. Kristensen, J. Hübner, W. Xu, and M. Bazylenko, Techn. Digest of OFC/IOOC '99, paper WM19, , 250 (1999).

${ }^{6}$ S. Kurimura and Y. Uesu, J. Appl. Phys. 81, 369 (1997).

${ }^{7}$ V. Kirilyuk, A. Kirilyuk, and Th. Rasing, Appl. Phys. Lett. 70, 2306 (1997).

${ }^{8}$ S. I. Bozhevolnyi, J. M. Hvam, K. Pedersen, F. Laurell, H. Karlsson, T. Skettrup, and M. Belmonte, Appl. Phys. Lett. 73, 1814 (1998).

${ }^{9}$ C. V. Poulsen, T. Storgaard-Larsen, J. Hübner, and O. Leistiko, Proc. SPIE 2998, 132 (1997).

${ }^{10}$ M. Svalgaard, C. V. Poulsen, A. Bjarklev, and O. Poulsen, Electron. Lett. 30, 1401 (1994).

${ }^{11}$ S. Kielich, IEEE J. Quantum Electron. 5, 562 (1969).

${ }^{12}$ J. M. Dell, M. J. Joyce, and G. O. Stone, Proc. SPIE 2289, 185 (1994).

${ }^{13}$ T. Fujiwara, D. Wong, Y. Zhao, S. Fleming, S. Poole, and M. Sceats, Electron. Lett. 31, 573 (1995). 